\title{
Endoscopic Diagnosis and Extraction of Gastric Parasites
}

\author{
M. Muñoz-Navas, E. Macías, L. García-Villarreal, J. Val, R. Angós \\ Endoscopic Unit, University Clinic of the University of Navarra \\ Pío XII, s/n 31008 Pamplona, Spain
}

The value of endoscopy in the diagnosis and extraction of parasites in the human digestive tract has been demonstrated (1). Worms attached to gastric mucosa are exceptional and only a small number of cases have been reported in the literature $(2,3)$. A 40-year-old male presented with epigastric pain of one month duration. The pain onset occurred immediately after returning from the mountains where the patient drank spring water. Physical examination and laboratory data were normal. Stool specimens were negative for parasites and culture. An abdominal ultrasound and upper gastrointestinal X-ray series were normal before consulting our hospital.

Because of persistent gastrointestinal symptoms an endoscopic examination was performed revealing the presence of a flat red-purple lesion of about $10 \mathrm{~mm}$ diameter on the posterior wall of the antrum from which a white, long and round structure of about $20 \mathrm{~mm}$ long by $1 \mathrm{~mm}$ width protruded. It showed a lively wriggling movement (Figure 1). After introducing a biopsy forceps we seized the parasite from the tail verifying it was firmly attached to the mucosa (Figure 2), and we proceeded to capture it. A biopsy specimen was taken from the antrum revealing chronic superficial gastritis.

The helminth was identified as the larva of a roundworm belonging to the Rabditoid family. It is a pseudoparasite only pathogenic after ingestion of raw vegetables or polluted water. The patient's symptoms improved after endoscopy and he refused any additional treatment.

The finding of worms attached to human gastric mucosa is exceptional because of the hostile atmosphere due to gastric acid $\mathrm{pH}$. The presence of gastric atrophy would increase the $\mathrm{pH}$ and so allow the parasite infestation but, in our case, the biopsy specimens showed a superficial gastritis that does not change intragastric $\mathrm{pH}$. Our case is the first one reported in a white European patient. We have found in the literature some papers reporting the penetration into the stomach wall by a larva of Anisakiasis in Oriental patients after eating raw fish $(2,4,5)$. Another paper has reported the diagnosis and extraction of Necator americanus in the stomach of a black patient (3). We presume that the transmission pathway was polluted water.

\section{REFERENCES}

1. Genta RM, Woods KL: Endoscopic diagnosis of hookworm infection. Gastroint. Endosc. 1991; 37: 476-478. 
2. Akasaka Y, Kizu M, Aoike A et al.: Endoscopic management of acute gastric anisakiasis. Endoscopy 1979; 2: 158-162.

3. Dumont A, Seferian V, Barbier P: Endoscopic discovery and capture of Necator americanus in the stomach. Endoscopy 1983; 15: 65-66.

4. Deardorff TL, Fukumura T, Raybourne RB: Invasive anisakiasis. A case report from Hawaii. Gastroenterology 1986; 90: 1047-1050.

5. Hsiu JG, Gamsey AJ, Ives CE et al.: Gastric anisakiasis: report of a case with clinical, endoscopic and histological findings. Am. J. Gastroenterol. 1986; 81: 1185-1187. 


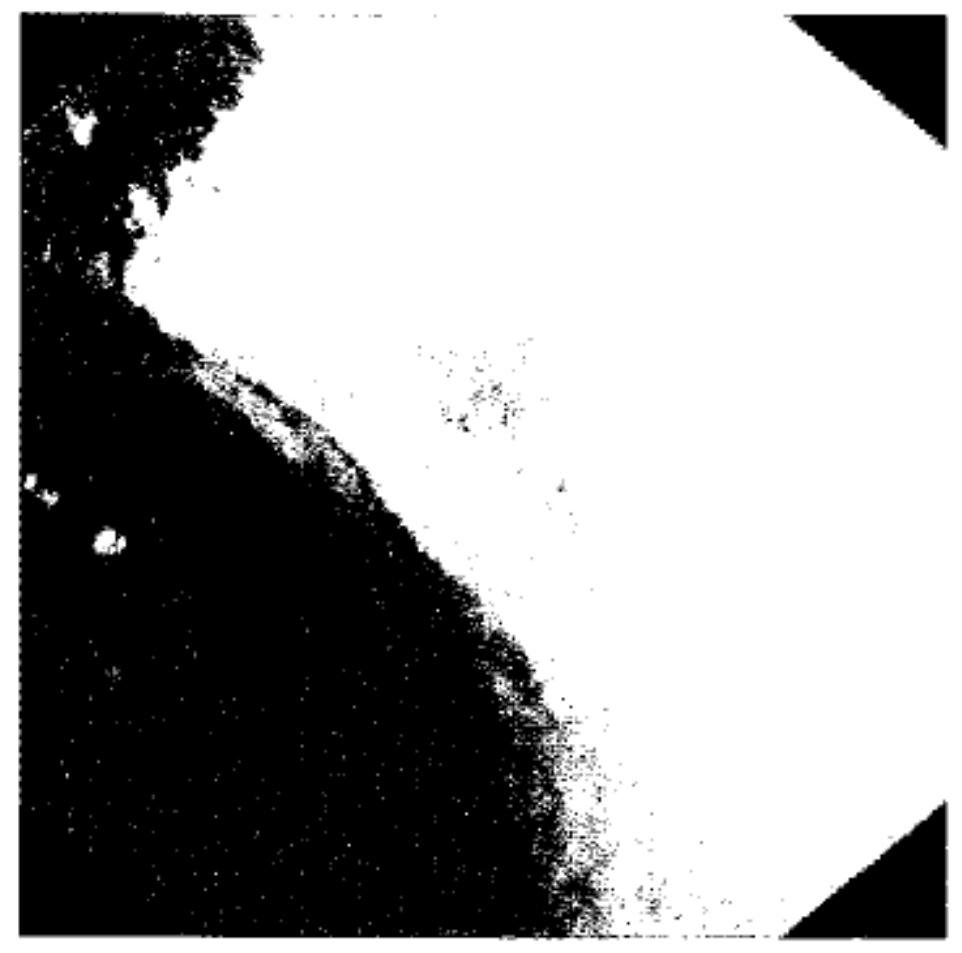

Figure 1. Endoscopic picture showing a living white roundworm on the posterior wall of the antrum.

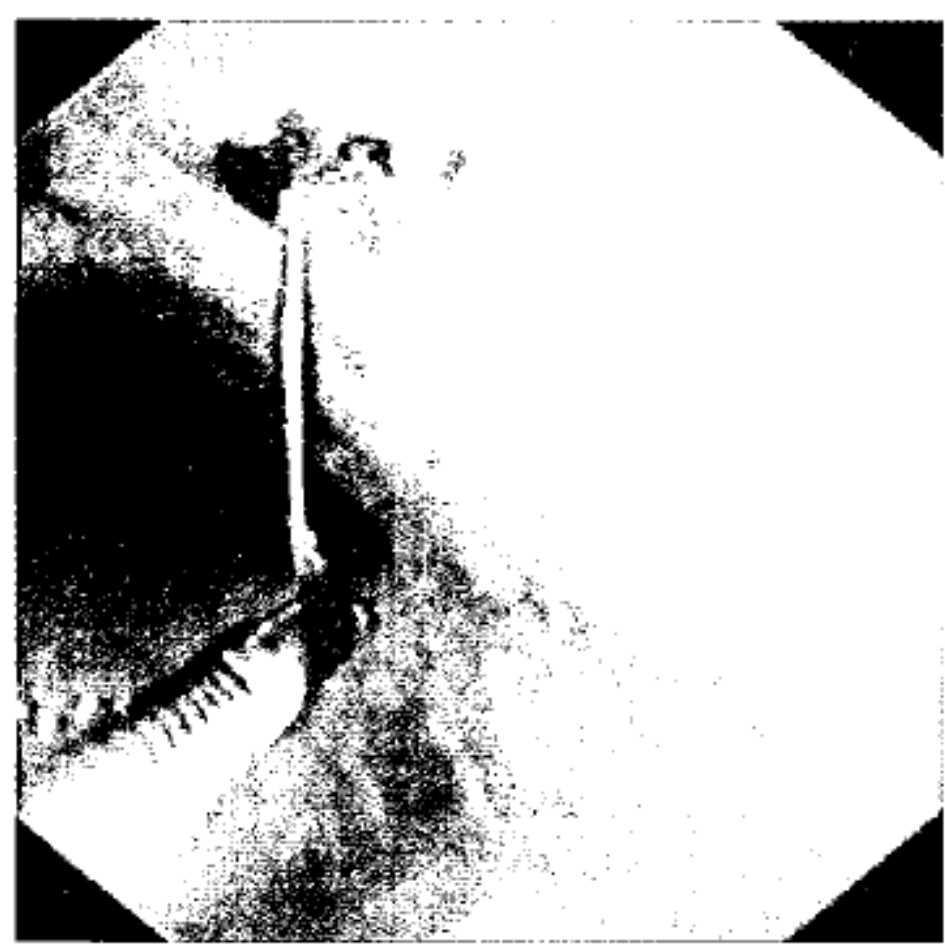

Figure 2. The parasite is caught with a biopsy forceps. 